\title{
Experimental evaluation of Hovenring Bridge stay-cable vibration
}

\author{
T. Argentini, L. Rosa \& A. Zasso \\ Politecnico di Milano, Department of Mechanical Engineering, Italy
}

\begin{abstract}
This paper deals with the experimental investigations carried out on a cable-stayed pedestrian and bicycle bridge. Shortly after the installation of the bridge, it turned out that the cables vibrated in the wind. Experimental investigations were soon started to determine the exact cause of the vibrations with the aim of finding a definitive solution. Vortex shedding was found to cause the vibrations and a Stockbridge damper was mounted to test its effectiveness. A case of vortex induced vibrations was also measured together with wind speed on site as a confirmation of the study. Finally, a prediction of the number of cycles suffered by the stays as a consequence of vortex shedding was also done in order to evaluate the cumulated stress and the residual fatigue life of the structural elements.

Keywords: vortex shedding, cable-stayed bridge, pedestrian bridge, experimental tests, fatigue.
\end{abstract}

\section{Introduction}

Cables are efficient structural elements that are used in cable-stayed bridges, suspension bridges and other cable structures. These structural elements are subject to a large number of environmental excitations. Wind is one of the principal and the most frequent among these and unfortunately tensioned strands of suspension bridges and cable-stayed bridges can suffer from a high level of vibration due to the wind-cable interaction [1]. There has been extensive research into this topic over the last decades and vortex shedding, wind-rain instability and dry-galloping are the typical causes of wind excitation; see, for example, 
[2-8]. In this work we will focus on vortex shedding induced vibrations. This type of vibration can induce excessive stresses that may lead, sometimes in a short period of time, to fatigue failures of the cables themselves as well as of the anchorages, and consequently threatens the safety and serviceability of the structures $[9,10]$. The parameter which mainly controls this phenomenon is the damping. Each cable or stay has a structural damping which defines its capacity the dissipate energy internally while vibrating. This material and frictional damping is usually quite low, so it is necessary to use dampers in order to successfully control the vibrations. The structural damping determines the range of frequency where vibration dampers may be needed. It cannot be calculated in advance so experimental tests are usually required to directly measure the damping on the real structure [11-13].

This paper deals with the experimental investigations carried out on a cablestayed pedestrian and bicycle bridge. Shortly after installation of the bridge, it turned out that the cables vibrated in the wind. Experimental investigations were soon started to determine the exact cause of the vibrations and with the aim of finding a solution; vortex shedding was found to cause the vibrations. A Stockbridge type damper was mounted to test its efficiency and visual tests after the installation of the damping device showed an almost total absence of vibrations [14]. The results in terms of natural frequency, modal deformation and modal damping (non-dimensional damping ratio $\xi$ ), allowed for the definition of a numerical model representative of the damping-frequency trend, to be used in finite element model (FEM) numerical simulations to predict the cables vortex induced response and to design the dampers and their most efficient position along the cable.

As a confirmation of the study a case of vortex induced vibrations was measured on a cable together with wind speed on site. Finally, a prediction of the number of cycles suffered by the stays as a consequence of the vortex shedding excitation was done in order to evaluate the cumulated stress and the residual fatigue life of the structural elements. A detailed discussion about the optimization of the FEM, the design of the dampers and the prediction of the cumulated stress on the fixing elements of the stays, will be presented in a future work.

\section{The bridge}

The Hovenring bridge, built in Eindhoven, is a bicycle roundabout flyover with a central $70 \mathrm{~m}$ high steel pylon carrying a $72 \mathrm{~m}$ diameter circular bridge deck suspended through 24 stay-cables; see Figure 1(a).

The 24 stays are $50 \mathrm{~mm}$ in diameter and are $53 \mathrm{~m}$ long: 8 cables are tensioned with a force of $T_{1}=705 \mathrm{kN}$, while the remaining 16 cables are tensioned with a force of $T_{2}=470 \mathrm{kN}$. The bridge deck is virtually floating over a motorway junction separating bicycles from cars. It is a signature bridge especially developed for this location. 


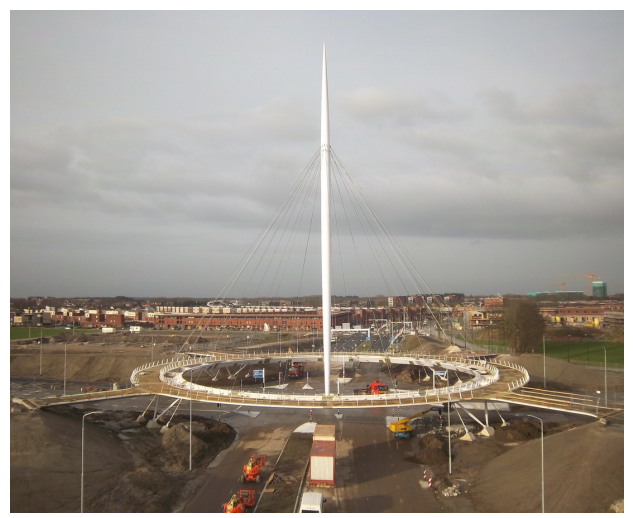

(a)

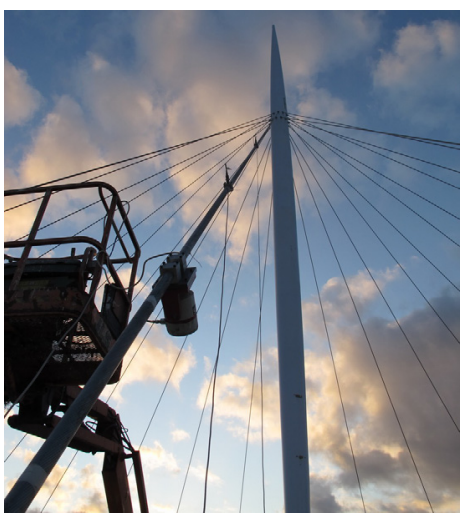

(b)

Figure 1: (a) The Hovering bridge. (b) The shaker and the servo-accelerometers positioned at different heights on the cable.

\section{Measurement set-up}

In-situ tests were carried out to experimentally assess the modal parameters of the cables and to test a suitable damper. Two kind of cables were analyzed: one high-tension cable $\left(T_{1}\right)$ and a low-tension cable $\left(T_{2}\right)$.

Modal parameters were estimated from the free vibration response of the cables. The natural frequency detection was made by imposing an initial forcing condition, obtained with an impulsive excitation, or with a random high-frequency motion.

The non-dimensional damping $\xi$ was estimated on single harmonic decay time histories. For each tested cable an initial condition of single harmonic motion was induced through a shaker fixed on the hanger, at one of the cable modal natural frequencies. Due to the low frequency, the first mode was manually excited. In this case the tests were repeated with and without the shaker mounted on the cable. The shaker has a mass of about $12 \mathrm{~kg}$ while the linear mass of the cable is $14.4 \mathrm{~kg} / \mathrm{m}$. The wide difference between the masses and the agreement of the results confirmed the consistency of the method adopted, highlighting that the shaker mounted on the cable did not affect the damping estimate.

When a steady condition of single mode vibration was reached, the shaker was turned off, letting the hanger move freely. The time history showing the decreasing of the oscillation amplitudes were recorded with two servo accelerometers fixed parallel to the excitation of the electromagnetic shaker in two different positions along the hanger: at $5 \mathrm{~m}$ and at $10 \mathrm{~m}$ from the socket. Figure 1(b) shows the shaker and the two accelerometers fixed to a stay. The wind speed was also measured on site with a cup-type anemometer placed $2.5 \mathrm{~m}$ from the deck level. 
Table 1: Estimate of cable damping and related vibration amplitudes.

\begin{tabular}{r|rrr|rrr}
\hline & \multicolumn{3}{|c|}{ High-tension cable $T_{1}$} & \multicolumn{3}{c}{ Low-tension cable $T_{2}$} \\
Mode & Freq (Hz) & $\xi(\%)$ & Ampl (mm) & Freq (Hz) & $\xi(\%)$ & Ampl (mm) \\
\hline 1 & 2.06 & 0.048 & 10.2 & 1.79 & 0.061 & 31.3 \\
2 & 4.11 & 0.036 & 4.2 & 3.58 & 0.051 & 7.5 \\
3 & 6.03 & - & - & 5.35 & - & - \\
4 & 7.99 & - & - & 7.07 & 0.025 & 0.4 \\
5 & 9.91 & - & - & 8.8 & 0.024 & 1.1 \\
6 & 11.82 & 0.013 & 0.3 & 10.49 & - & - \\
7 & 13.85 & 0.011 & 0.2 & 12.24 & 0.024 & 0.3 \\
8 & 15.88 & 0.013 & 0.1 & 14.06 & 0.043 & 0.2 \\
9 & 18.01 & 0.011 & 0.2 & 15.93 & 0.056 & 0.2 \\
\hline
\end{tabular}

\section{Results}

\subsection{Cable modal parameters}

Table 1 shows the experimental frequencies and damping coefficients evaluated from the experimental tests. The column "Ampl"is quoting the mean amplitude of oscillation of the antinode of vibration during free decay response. Great care has been taken to avoid conditions in which the cable could be affected by wind induced excitation, withdrawing the tests during periods when wind excitation occurred. For this reason the missing data (-) are not reported because it was not possible, during the test days, to reliably measure structural damping due to vortex shedding excitation by the natural wind.

Two examples of signal analysis used to estimate the modal parameters are reported in the following figures: the first mode (Figure 2) and the eighth mode (Figure 3) of the high-tension cable. Figures 2(a) and 3(a) show the time history of free decay response of one accelerometer: the signals are solid and are not affected by relevant noise. Figures 2(b) and 3(b) show the Hilbert transform in logarithmic scale (magnitude) used to estimate the damping coefficient $\xi$, which is proportional to the slope of the curve as a function of time. One can see these trends are fairly linear, indicating that the damping does not change in the decay time, that is with the vibration amplitude.

Figure 4(a) shows the spectra of the previous time histories: it is possible to note that the harmonic layout of each channel is single-harmonic, proving that only one modal shape provides the overall motion. In this case it ensures that the non-dimensional damping computed is related only to the single modal shape.

Figure 4(b) shows the non-dimensional damping $\xi$ plotted versus the relative frequency values. It can be noticed that the measured damping coefficients of the high-tension cable are very low and generally decrease with the frequency. On the 


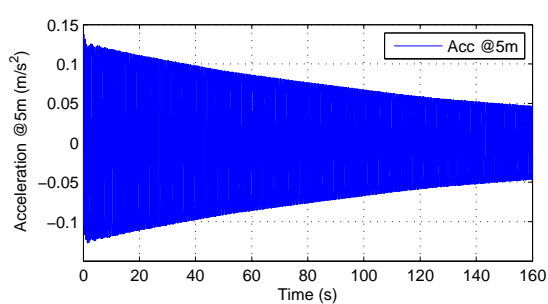

(a)



(b)

Figure 2: High tension cable, first mode. (a) Time history of free decay response of Accelerometer @5m. (b) Magnitude of Hilbert transform and linear interpolation.



(a)

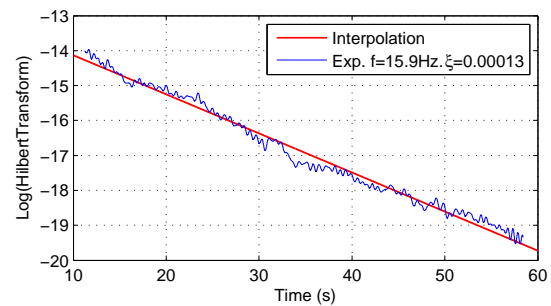

(b)

Figure 3: High tension cable, eighth mode. (a) Time history of free decay response of Accelerometer @10m. (b) Magnitude of Hilbert transform and linear interpolation.

contrary the other cable shows an oscillating trend: in this case the lower tension makes the stay more sensible to the boundary condition. This behaviour could also be associated with a larger contribution of the whole structure to the global damping at some specific frequencies.

\subsection{Tuned mass damper visual observations}

During the experimental stage a Stockbridge tuned mass damper was mounted on a cable to assess its effectiveness in suppressing vortex shedding excitations. Visual observations confirmed that this kind of damping device provides the necessary amount of damping to avoid vibrations.

The results in terms of natural frequency, modal deformation and modal damping (non-dimensional damping ratio $\xi$ ), allowed for the definition of numerical model representations of the damping-frequency trend, to be used in a very detailed finite element model. This FEM developed with the data acquired 


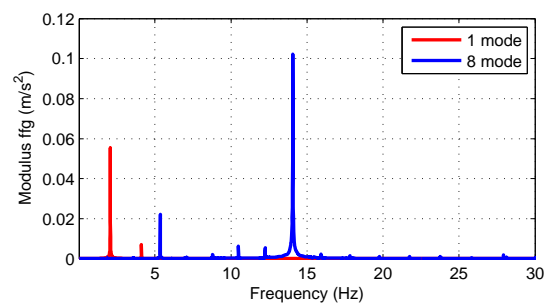

(a)

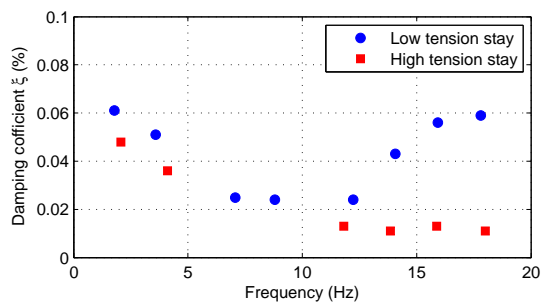

(b)

Figure 4: High tension cable, 1st and 8th mode. (a) Magnitude of the spectrum of free decay response. (b) Measured damping coefficients $(\xi, \%)$ as a function of frequency.

during the experimental tests will be used to design the characteristic of the dampers and their position along the cables.

\subsection{Vortex shedding observation}

During the experimental stage, one of the instrumented cables experienced wind induced vibrations and it was possible to record the cable response. In particular, the response of the accelerometer @5m was measured, together with the anemometer. Luckily this observed event of wind vortex shedding excitation showed the cable quite perfectly oriented "cross wind", which is the most severe condition for vortex induced vibrations (Figure 6(b)). Furthermore the cable oscillations were quite perfectly oriented in the direction of the accelerometers with negligible component in the other orthogonal direction.

Another important parameter with the vortex shedding is the Scruton number, defined as:

$$
S c=\frac{m \xi}{\rho D^{2}}
$$

where $m$ is the mass of cable per unit length, $x i$ the damping ratio, $\rho$ the air density and $D$ the cable outside diameter. The higher this number is the better since vortex shedding oscillations become smaller as the Scruton number is increased. Furthermore, this number should be greater than 10 to avoid rainwind oscillations [13]. In the present case $0.7<S c<2$, depending on the frequency considered, so vortex shedding oscillations are very likely. Figure 5(a) shows the time history of the recorded acceleration. It can be noticed that a quasi-steady response was measured. Looking at the zoomed time history, we can see that a prevailing mono-harmonic response is present. This is confirmed by Figure 5(b) which shows the magnitude of the spectrum of the recorded acceleration: a peak at $10.94 \mathrm{~Hz}$ with amplitude $0.75 \mathrm{~g}$ is present, corresponding to an oscillation amplitude of about $1.6 \mathrm{~mm}$. Figure 6(a) shows the wind speed 


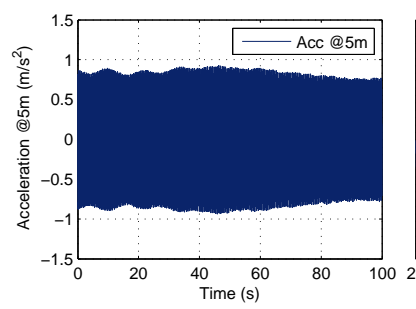

(a)

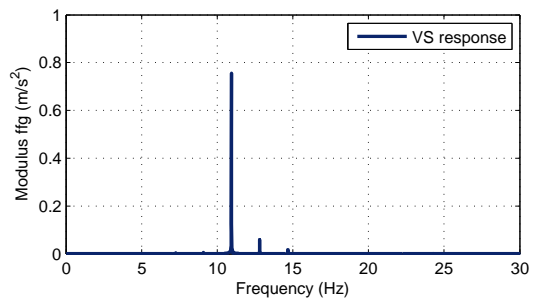

(b)

Figure 5: Vortex shedding response. (a) Recorded acceleration of accelerometer @ $5 \mathrm{~m}$. (b) Spectrum of the acceleration.

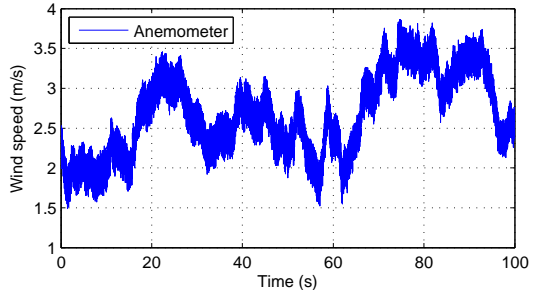

(a)

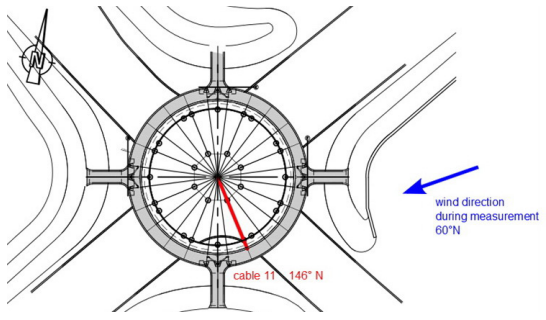

(b)

Figure 6: Vortex shedding response. (a) On-site wind speed measurements. (b) Sketch of wind angle and cable 11, during vortex shedding observations.

recorded simultaneously with the acceleration: a mean value of $2.6 \mathrm{~m} / \mathrm{s}$ is measured.

The wind speed necessary to have a shedding frequency $f=10.94 \mathrm{~Hz}$, considering a Strouhal number $S t=0.2$ and a cable diameter $d=0.050 \mathrm{~m}$ would be:

$$
V=\frac{f d}{S t}=2.73 \quad(\mathrm{~m} / \mathrm{s})
$$

which is compatible with the velocity measured on site.

\subsection{Prediction of the number of cycles}

To collect the data necessary for the fatigue assessment a prediction of the number of cycles suffered by the stays as a consequence of vortex shedding excitation was done. The analysis considered the lapse of time from the bridge installation until the vibrations were stopped by application of temporary braces between cables. This period is referred to by the acronym CBO (Cable Vibration On).

The calculation of the wind speed at the site, necessary for the determination of the number of cycles, was done using the wind data recorded at Eindhoven 


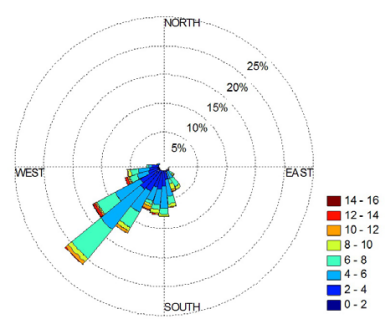

(a)

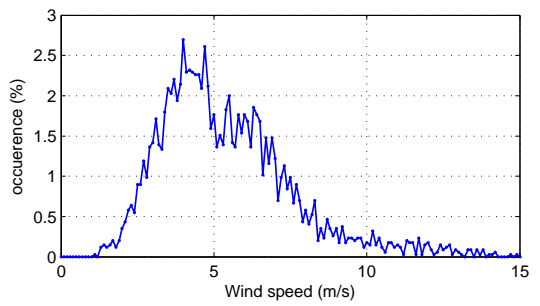

(b)

Figure 7: (a) Wind rose during period CVO (at Eindhoven airport $10 \mathrm{~m} \mathrm{AMSL):}$ $10 \mathrm{~min}$ wind speed in $(\mathrm{m} / \mathrm{s})$. (b) Wind speed distribution.

airport during $\mathrm{CBO}$, summarized in Figures 7(a) and 7(b). The first figure shows that during that period the prevailing winds were from the $\mathrm{SW}$; the second one shows the wind speed distribution divided in intervals of $0.1 \mathrm{~m} / \mathrm{s}$.

The measurements performed with the cup-anemometer placed $2.5 \mathrm{~m}$ above deck height during the experimental activities were used to built a statistical relation between the wind at the airport and at the bridge site. The idea was to calculate the ratio $\gamma$ between the mean speeds at Hovenring registered during the experimental test days and the 10 minutes airport wind speed during the $\mathrm{CBO}$. This ratio obtained from the elaboration of the wind histories does not exhibit, however, a regular trend or mean value, so the calculation of the wind speed at Hovenring was attempted using two different scaling factors: $\gamma=1$ and $\gamma=0.6$.

The prediction of the number of cycles during CBO was made with the following assumptions:

- The wind speed profile of the atmospheric boundary layer was neglected and a uniform wind speed profile was adopted. This hypothesis is conservative since in reality the shedding frequency changes along the cable height, therefore shedding forcing is not uniform along the cable length.

- Turbulence was neglected and a laminar flow was assumed. This hypothesis is conservative since turbulence attenuates vortex shedding induced vibration.

- Steady-state oscillations were considered. In reality, there is a build-up time necessary to reach the maximum amplitude of vibration that may last several minutes.

- Wind direction was neglected. The angle of incidence between mean wind speed and cable orientation is however an important parameter. It is expected that cables in cross-flow suffer most from vortex induced vibrations.

- For each mode, the lock-in region was selected between $0.9<V / V_{S t}<1.3$, being $V_{S t}=f_{i} d / 0.2, f_{i}$ the mode frequency and $d$ the cable diameter. In this range of velocities, we expect that the maximum vibration amplitude can be reached for each mode. 
Table 2: Estimated number of cycles: $\gamma=1$ and $\gamma=0.6$.

\begin{tabular}{c|c|ccc|cc|cc}
\hline Mode & $\begin{array}{c}\text { Freq } \\
(\mathrm{Hz})\end{array}$ & $\begin{array}{c}V_{\min } \\
(\mathrm{m} / \mathrm{s})\end{array}$ & $\begin{array}{c}V_{S t} \\
(\mathrm{~m} / \mathrm{s})\end{array}$ & $\begin{array}{c}V_{\max } \\
(\mathrm{m} / \mathrm{s})\end{array}$ & $\begin{array}{c}\gamma=1 \\
(\text { hours })\end{array}$ & $\begin{array}{c}\gamma=1 \\
(\text { cycles })\end{array}$ & $\begin{array}{c}\gamma=0.6 \\
\text { (hours) }\end{array}$ & $\begin{array}{c}\gamma 0.6 \\
\text { (cycles) }\end{array}$ \\
\hline 1 & 2.1 & 0.5 & 0.5 & 0.7 & 0 & 0 & 0.1 & $1.11 \mathrm{E} 03$ \\
2 & 4.3 & 1 & 1.1 & 1.4 & 1.5 & $2.26 \mathrm{E} 04$ & 16.2 & $2.51 \mathrm{E} 05$ \\
3 & 6.4 & 1.4 & 1.6 & 2.1 & 8.6 & $1.98 \mathrm{E} 06$ & 81.8 & $1.89 \mathrm{E} 06$ \\
4 & 8.6 & 1.9 & 2.1 & 2.8 & 33.8 & $1.05 \mathrm{E} 06$ & 178.4 & $5.52 \mathrm{E} 06$ \\
5 & 10.7 & 2.4 & 2.7 & 3.5 & 78.6 & $3.03 \mathrm{E} 06$ & 193.5 & $7.45 \mathrm{E} 06$ \\
6 & 12.9 & 2.9 & 3.2 & 4.2 & 134.5 & $6.24 \mathrm{E} 06$ & 181.7 & $8.44 \mathrm{E} 06$ \\
7 & 15 & 3.4 & 3.8 & 4.9 & 174.7 & $9.43 \mathrm{E} 06$ & 157.9 & $8.53 \mathrm{E} 06$ \\
8 & 17.2 & 3.9 & 4.3 & 5.6 & 180.8 & $1.12 \mathrm{E} 07$ & 104.3 & $6.46 \mathrm{E} 06$ \\
9 & 19.4 & 4.4 & 4.8 & 6.3 & 178.8 & $1.25 \mathrm{E} 07$ & 66.2 & $4.62 \mathrm{E} 06$ \\
10 & 21.5 & 4.8 & 5.4 & 7 & 181.7 & $1.40 \mathrm{E} 07$ & 45.9 & $3.56 \mathrm{E} 06$ \\
\hline
\end{tabular}

Considering these hypotheses, it is expected that the number of cycles and the related stress will be overestimated, but conservative. For each mode the following procedure is adopted to estimate the number of cycles:

- The lock-in region is identified $\left(0.9<V / V_{S t}<1.3\right)$.

- The percentage of occurrence of the lock-in region during CBO is computed from airport data using the scaling factor $\gamma$ and the corresponding number of hours is computed.

- The number of cycles is computed knowing the modal frequency and the number of hours.

Table 2 shows the number of cycles estimated. One can see that the number is quite large, indicating that a detailed fatigue study aimed at the estimation of the cumulated stress due to vortex shedding excitation is necessary in order to evaluate the residual fatigue life of the most sensible structural elements. This study will be presented in a future work together with the FEM analysis.

\section{Conclusions}

The measuring experiments on the Hovenring bridge allowed us to evaluate the real non-dimensional damping of the stays as a function of the modal frequency, with reference to a set of different tension cables. The experimental evaluation of the stay damping on the real structure allowed us to take into account the contribution of the end fittings and connections to the structure increasing the global damping with respect to the cables alone.

The results in terms of natural frequency, modal deformation and modal damping, allowed for the definition of numerical model representatives of the damping-frequency trend, to be used in finite element model (FEM) numerical 
simulations to predict the cables vortex induced response and to test the efficacy of the dampers before their installation.

A prediction of the number of cycles suffered by the stays as a consequence of vortex shedding was also done. This information will be very important in the next step of the work, aimed to evaluate the cumulated stress and the related residual fatigue life of the most sensible elements. The final target of this study will be the decision on the replacement of these elements.

The present work confirms that vortex shedding should be considered in the design stage, and that it can be dangerous and uneconomic to consider a solution against vortex shedding after the completion of a structure.

\section{References}

[1] Zasso, A., Belloli, M., Giappino, S. \& Muggiasca, S., Pressure field analysis on oscillating circular cylinder. Journal of Fluids and Structures, 24(5), pp. 628-650, 2008.

[2] Chen, W., Li, H., Ou, J. \& Li, F., Numerical simulation of vortex-induced vibrations of inclined cables under different wind profiles. Journal of Bridge Engineering, 18(1), pp. 42-53, 2013.

[3] Belloli, M., Giappino, S., Muggiasca, S. \& Zasso, A., Force and wake analysis on a single circular cylinder subjected to vortex induced vibrations at high mass ratio and high Reynolds number. Journal of Wind Engineering and Industrial Aerodynamics, 103, pp. 96-106, 2012.

[4] Zuo, D. \& Jones, N.P., Interpretation of field observations of wind- and rain-wind-induced stay cable vibrations. Journal of Wind Engineering and Industrial Aerodynamics, 98(2), pp. 73-87, 2010.

[5] Matsumoto, M., Yagi, T., Hatsuda, H., Shima, T., Tanaka, M. \& Naito, H., Dry galloping characteristics and its mechanism of inclined/yawed cables. Journal of Wind Engineering and Industrial Aerodynamics, 98(6-7), pp. 317 327, 2010. 0167-6105.

[6] Malavasi, S., Corretto, R., Belloli, M., Giappino, S. \& Muggiasca, S., Wake visualization and pressure field analysis on an oscillating cylinder. 5th European and African Conference on Wind Engineering, EACWE 5, Proceedings, 2009.

[7] Macdonald, J.H.G. \& Larose, G.L., Two-degree-of-freedom inclined cable galloping-part 1: General formulation and solution for perfectly tuned system. Journal of Wind Engineering and Industrial Aerodynamics, 96(3), pp. 291-307, 2008.

[8] Belloli, M., Diana, G., Resta, F. \& Muggiasca, S., A numerical model to reproduce vortex induced vibrations of a circular cylinder. American Society of Mechanical Engineers, Pressure Vessels and Piping Division (Publication) PVP, volume 2006, 2006.

[9] Boniardi, M., Diana, G. \& Tagliabue, C., Fretting-fatigue phenomena on an electrical conductor. Fenomeni di fretting-fatigue su un conduttore elettrico, 99(11-12), pp. 15-24, 2007. 
[10] Cluni, F., Gusella, V. \& Ubertini, F., A parametric investigation of windinduced cable fatigue. Engineering Structures, 29(11), pp. 3094-3105, 2007.

[11] Argentini, T., Belloli, M., Rosa, L., Sabbioni, E., Zasso, A. \& Villani, M., Modal identification of a cable-stayed bridge by means of truck induced vibrations. Conference Proceedings of the Society for Experimental Mechanics Series, Jacksonville, Florida, volume 1, pp. 165-172, 2012.

[12] Belloli, M., Rosa, L., Villani, M. \& Zasso, A., Full scale measurements to determine the self-damping of stays. EVACES '11, eds. C. Gentile \& F. Benedettini, Starrlink Editrice, Brescia: Varenna, volume 2, pp. 439-446, 2011.

[13] Chan, J., Havard, D., Rawlins, C. \& Weisel, J., EPRI transmission line reference book: wind-induced conductor motion. Palo Alto, 2006.

[14] Diana, G., Cigada, A., Belloli, M. \& Vanali, M., Stockbridge-type damper effectiveness evaluation: Part i - comparison between tests on span and on the shaker. IEEE Transactions on Power Delivery, 18(4), pp. 1462-1469, 2003. 